\title{
A Variational Level Set Approach for Surface Area Minimization of Triply Periodic Surfaces
}

\author{
Y. Jung ${ }^{\mathrm{ab}}$, K. T. $\mathrm{Chu}^{\mathrm{c}}$, and S. Torquato ${ }^{\text {abde }}$ \\ aPrinceton Institute for the Science and Technology of Materials, Princeton \\ University, Princeton, New Jersey 08544 \\ ${ }^{\mathrm{b}}$ Department of Chemistry, Princeton University, Princeton, New Jersey 08544 \\ ${ }^{\mathrm{c}}$ Department of Mechanical and Aerospace Engineering, Princeton University, \\ Princeton, New Jersey 08544 \\ ${ }^{\mathrm{d} P r o g r a m}$ in Applied and Computational Mathematics, Princeton University, \\ Princeton, New Jersey 08544 \\ ${ }^{e}$ Princeton Center for Theoretical Physics, Princeton University, Princeton, New \\ Jersey 08544
}

In this paper, we study triply periodic surfaces with minimal surface area under a constraint in the volume fraction of the regions (phases) that the surface separates. Using a variational level set method formulation, we present a theoretical characterization of and a numerical algorithm for computing these surfaces. We use our theoretical and computational formulation to study the optimality of the Schwartz P, Schwartz D, and Schoen G surfaces when the volume fractions of the two phases are equal and explore the properties of optimal structures when the volume fractions of the two phases not equal. Due to the computational cost of the fully, threedimensional shape optimization problem, we implement our numerical simulations using a parallel level set method software package.

\section{Introduction}

Triply periodic minimal surfaces [1, 2, 3] are objects of great interest to physical scientists, biologists, and mathematicians because they naturally arise in a variety of systems, including block copolymers [4, nanocomposites [5], micellar materials [ 6], and lipid-water systems and certain cell membranes [3, 7, 8, 9. There are two key feature of these surfaces: (1) the mean curvature $^{1}$ is zero everywhere on the

\footnotetext{
${ }^{1}$ The mean curvature, $H(\mathbf{x})$, at a point $\mathbf{x}$ of a surface is the average of the two principal normal curvatures, $\kappa_{1}(\mathbf{x})$ and $\kappa_{2}(\mathbf{x}): H(\mathbf{x})=\frac{1}{2}\left(\kappa_{1}(\mathbf{x})+\kappa_{2}(\mathbf{x})\right) . H(\mathbf{x})$ is also conveniently represented in
} 
surface and (2) they are periodic in all three coordinate directions (i.e. they extend infinitely in all directions and possess the symmetry of one of the crystallographic space groups). An important subclass of triply periodic minimal surfaces are those that partition space into two disjoint but intertwining regions that are simultaneously continuous [10]. Examples of such surfaces include the Schwartz primitive (P), the Schwartz diamond (D), and the Schoen gyroid (G) surfaces (see Figure 1).
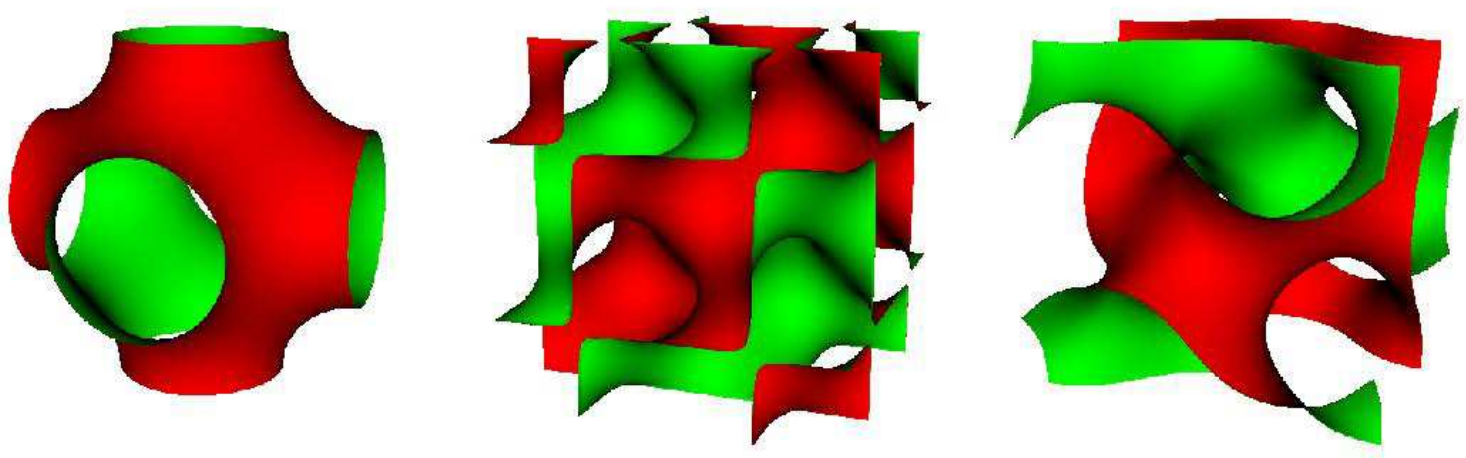

Figure 1. Unit cell for three common minimal surfaces: Schwartz P surface (left), Schwartz D surface (middle), and Schoen G surface (right).

Recently, there has been a resurgence of interest in triply periodic minimal surfaces because two-phase composites whose phases are separated by such surfaces have been shown to be optimal with respect to multifunctional optimization of their material properties [ 11, 12, 13]. For instance, it has been shown that maximal values for the sum of the effective thermal and electrical conductivities is achieved by two-phase structures separated by Schwartz P and Schwartz D surfaces [11, 12, These structures have also been discovered to be optimal for multifunctional bulk modulus and electrical conductivity optimizations [13]. As another example, the porous medium with the Schwartz $\mathrm{P}$ interface was found (via computer simulation) to have the largest fluid permeability over a range of microstructures examined [14].

In [14], Jung and Torquato observed that the fluid permeabilities of porous, bicontinuous microstructures is inversely related to the total interfacial surface area per unit volume. This observation led them to conjecture that the maximal fluid permeability for a triply periodic porous medium is achieved by the structure that minimizes the total interfacial area [14]. One of the most interesting aspects of this conjecture is its focus on a global property of the interface (i.e., total surface area) rather than on local (e.g., differential) properties of the surface (e.g., mean curvature).

terms of the divergence of the unit normal vector (or equivalently, the trace of the gradient of $\mathbf{n}$ ): $H(\mathbf{x})=-\frac{1}{2} \nabla \cdot \mathbf{n}=-\frac{1}{2} \operatorname{tr}(\operatorname{grad} \mathbf{n})$. 
Unfortunately, exploration of global properties of surfaces, such as the total surface area, seems to have received little attention in the literature. Classically, the study of surfaces and their properties has been the realm of differential geometry. In that field, research has traditionally focused on characterization and examination of minimal surfaces (i.e., surfaces with zero mean curvature). The search for minimal surfaces has been ongoing activity since the mathematician H. A. Schwartz published the first example of a minimal surface with full three-dimensional periodicity in 1865 [15]. A systematic exploration of triply periodic surfaces with nonzero mean curvature appears to have only been carried out relatively recently by Anderson, Davis, Scriven and Nitsche [10]. In their work, Anderson et al. numerically computed surfaces of prescribed mean curvature (possibly non-constant) and studied their properties.

In this paper, we consider triply periodic surfaces of nonzero mean curvature from a slightly different, but complementary, perspective. Motivated by the fluid permeability conjecture and the scarcity of mathematical results on surfaces of nonzero mean curvature, we examine triply periodic surfaces that minimize the total interfacial surface area while satisfying a constraint on the volume fractions of the regions that the surface separates. Interestingly, we find that the optimal surfaces for this problem are precisely those possessing a constant mean curvature. This result allows us to reproduce many of the results obtained by Andersen et al.. However, we emphasize that our approach comes from a fundamentally different perspective. Whereas Andersen et al. prescribe the mean curvature and consider properties of the resulting surface, we specify the volume fraction and ask what the mean curvature of the surface must be to minimize the total surface area. In general terms, we are interested in the properties of structures that arise when global geometric properties of a surface are prescribed (as opposed to examining the consequences of local properties).

The main conceptual foundations for our work come from the fundamental ideas underlying the level set method [16, 17]. Using these ideas, we have developed a novel numerical method for computing surfaces for which the surface area is a local minimum and that satisfy a specified volume fraction constraint. The ideas underlying the level method were also used to provide a theoretical characterization of the surfaces that solve of our constrained optimization problem. For both computational and theoretical purposes, the level set method formulation is convenient because it does not require explicit representation of the surface. From a theoretical perspective, the level set method also has the advantage that the formulas for key geometric quantities, such as the surface area and volume, have a relatively simple form.

In section 2, we begin by developing a level set formulation for describing and analyzing triply periodic surfaces. In section 3. we use this framework to theoretically characterize extrema of the total interfacial area under a constraint on the volume fraction of the phases. In section 4 , we present a numerical optimization procedure for computing surfaces that are local minima of the total interfacial area subject to a volume constraint. Finally, in section [5, we use a parallel implementation of 
our numerical algorithm to study several physically and mathematically interesting surfaces. Parallelism was utilized to help deal with the computational cost of our fully three-dimensional optimization problems.

\section{Level Set Formulation}

Our exploration of triply-periodic surfaces that are local minima of the total interfacial area subject to a volume constraint is based on ideas underlying the application of the level set method to shape optimization [18, 19]. Following the usual methodology [18, 19, we represent surfaces as the zero level set of an embedding function, $\phi(x)$, defined throughout the three-dimensional volume and use variational calculus to derive the relationship between $\phi$ and global geometric properties of the surface.

In this section, we present a detailed discussion of the level set formulation for surface area minimization in the presence of a volume fraction constraint. While it is clear that similar formulations have been used in previous studies [ 19], the explicit formulas and derivation for the most important geometric quantities were not provided. In the present discussion, which is intended to fill this apparent gap in the literature, we derive formulas for the area and volume of a triply-periodic surface and the variations of these quantities with respect to $\phi$. It is worth mentioning that the following derivation also holds for non-periodic surfaces and is easily extended to codimension-one surfaces in $n$-dimensional Euclidean space.

For the triply periodic problems that we are interested in, the domain is taken to be a unit cell of the periodically repeating structure. Let the surface of interest, $\Gamma$, be represented by the zero level set of $\phi$. Then $\Gamma$ divides the unit cell into two distinct phases. Without loss of generality, we define the region where $\phi(\mathbf{x})<0$ to be phase 1 (see Figures 2 and 33). An important assumption in our work is that a shift of the unit cell by a lattice vector does not cause an interchange of the phases. In this paper, systems possessing this property will be called phase-periodic.

In terms of the embedding function, $\phi$, our minimization problem may be stated as

$$
\text { minimize } \mathcal{A}(\phi) \text { subject to } f(\phi)=f_{o} \text {, }
$$

where $\mathcal{A}(\phi)$ is the total surface area of the zero level set, $f(\phi)$ is the volume fraction of phase 1 , and $f_{o}$ is the desired volume fraction for phase 1. Unfortunately, there are currently no theoretical results concerning the global minimum of our problem. However, as we shall see, there are many local minima for this problem. Because many interesting surfaces arise as local minima, we shall focus our attention on understanding the structure of these surfaces and neglect, for the moment, the question of global minimality.

We use the method of Lagrange multipliers to solve the optimization problem (11). The Lagrangian is given by

$$
\mathcal{L}(\phi, \lambda)=\mathcal{A}(\phi)+\lambda\left(f(\phi)-f_{o}\right),
$$




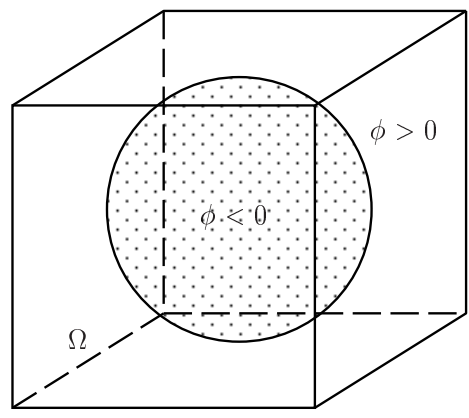

Figure 2. Schematic diagram of a codimension-one surface and the sign of the level set function, $\phi$, in various regions of the domain. The shaded and unshaded regions correspond to phase 1 and phase 2, respectively.
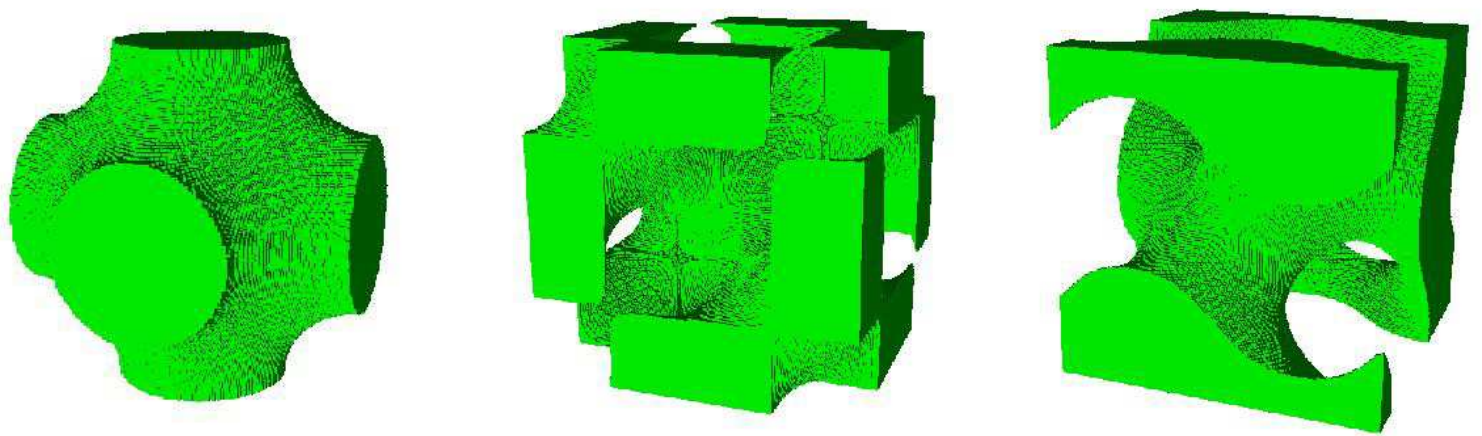

Figure 3. The phase 1 region corresponding to three different choices for $\Gamma$ : Schwartz P surface (left), Schwartz D surface (middle), and Schoen G surface (right).

where $\lambda$ is the Lagrange multiplier. Taking the variation of $\mathcal{L}$ with respect to $\phi$, we find that a necessary condition for a minimizer is

$$
\delta \mathcal{L}(\phi, \lambda)=\delta \mathcal{A}(\phi)+\lambda \delta f(\phi)=0 .
$$

This condition, together with the volume fraction constraint

$$
f(\phi)-f_{o}=0
$$

allows us to compute $\phi$ and $\lambda$.

\subsection{Volume integral formulation of surface integrals}

To compute the variations of $\mathcal{A}$ and $f$ required by our Lagrangian formulation of the optimization problem, it is convenient to first derive a relation that allows us to 
convert between surface integrals over $\Gamma$ and volume integrals over entire unit cell. Let $\Omega$ denote the entire unit cell, and let $\mathbf{N}$ denote an outward pointing unit normal vector on the boundary of the unit cell, $\partial \Omega$. Similarly, let $\Omega_{1}$ and $\mathbf{n}$ denote the region corresponding to phase 1 and an outward pointing normal vector on $\Gamma=\partial \Omega_{1}$. Now, consider the surface integral of an arbitrary function $p(\phi)$ over $\Gamma$ :

$$
\int_{\Gamma} p(\phi) d S
$$

Applying the divergence theorem, we can rewrite this surface integral as a volume integral over phase 1 and surface integrals over the intersections of phase 1 with the boundaries of the unit cell:

$$
\begin{aligned}
\int_{\Gamma} p(\phi) d S & =\int_{\Gamma} p(\phi) \mathbf{n} \cdot \mathbf{n} d S=\int_{\Gamma}\left(p(\phi) \frac{\nabla \phi}{\|\nabla \phi\|}\right) \cdot \mathbf{n} d S \\
& =\int_{\Omega_{1}} \nabla \cdot\left(p(\phi) \frac{\nabla \phi}{\|\nabla \phi\|}\right) d V-\int_{B}\left(p(\phi) \frac{\nabla \phi}{\|\nabla \phi\|}\right) \cdot \mathbf{N} d S
\end{aligned}
$$

where $B$ is the union of the intersections of phase 1 with the boundaries of the unit cell and we have identified $\mathbf{n}$ with $\frac{\nabla \phi}{\|\nabla \phi\|}$. Notice that the last surface integral term vanishes because we have assumed that $\phi$ is phase-periodic which means that $\left(p(\phi) \frac{\nabla \phi}{\|\nabla \phi\|}\right)$ is invariant under a translation of $\mathbf{x}$ by any lattice vector. Using phaseperiodicity, we can cancel out contributions of surface integrals from opposite faces of the unit cell because $\mathbf{N}$ on opposite faces have opposite sign.

Next, we observe that the volume integral over phase 1 can be rewritten as a volume integral over the entire unit cell:

$$
\int_{\Omega_{1}} \nabla \cdot\left(p(\phi) \frac{\nabla \phi}{\|\nabla \phi\|}\right) d V=\int_{\Omega}(1-\Theta(\phi)) \nabla \cdot\left(p(\phi) \frac{\nabla \phi}{\|\nabla \phi\|}\right) d V
$$

where $\Theta(\phi)$ is the Heaviside function

$$
\Theta(\phi)= \begin{cases}1 & \phi \geq 0 \\ 0 & \phi<0\end{cases}
$$

This expression can be further simplified via an integration by parts procedure to obtain:

$$
\begin{aligned}
\int_{\Gamma} p(\phi) d S= & -\int_{\Omega} \nabla(1-\Theta(\phi)) \cdot\left(p(\phi) \frac{\nabla \phi}{\|\nabla \phi\|}\right) d V \\
& +\int_{\partial \Omega}(1-\Theta(\phi))\left(p(\phi) \frac{\nabla \phi}{\|\nabla \phi\|}\right) \cdot \mathbf{N} d S \\
= & \int_{\Omega} \delta(\phi) \nabla \phi \cdot\left(p(\phi) \frac{\nabla \phi}{\|\nabla \phi\|}\right) d V \\
= & \int_{\Omega} p(\phi) \delta(\phi)\|\nabla \phi\| d V .
\end{aligned}
$$


where $\delta(\phi)$ is the Dirac delta function and we have eliminated the surface integral contribution by appealing to a similar line of reasoning as used when eliminating the surface integral term from (6). The form of (8) is not unexpected - the delta function ensures that only values of $p(\phi)$ on the surface $\Gamma$ contribute to the integral and the $\|\nabla \phi\|$ is the Jacobian that arises when $\phi$ (as opposed to a spatial coordinate in the direction normal to the surface) is used as the argument of the delta function. Equation (8) forms the foundation for much of the following mathematical development.

\subsection{Variation of the surface area}

Using the formula for the area of $\Gamma, \mathcal{A}(\phi)=\int_{\Gamma} d S$, and (8), we find that

$$
\mathcal{A}(\phi)=\int_{\Omega} \delta(\phi)\|\nabla \phi\| d V
$$

Taking the variation of this expression with respect to $\phi$ yields

$$
\begin{aligned}
\delta \mathcal{A}(\phi) & =\int_{\Omega}[\delta\{\delta(\phi)\}\|\nabla \phi\|+\delta(\phi) \delta\|\nabla \phi\|] d V \\
& =\int_{\Omega}\left[\delta^{\prime}(\phi)\|\nabla \phi\| \delta \phi+\delta(\phi) \frac{\nabla \phi \cdot \nabla(\delta \phi)}{\|\nabla \phi\|}\right] d V \\
& =\int_{\Omega}\left[\nabla(\delta(\phi)) \cdot \frac{\nabla \phi}{\|\nabla \phi\|} \delta \phi+\delta(\phi) \frac{\nabla \phi \cdot \nabla(\delta \phi)}{\|\nabla \phi\|}\right] d V
\end{aligned}
$$

where $\delta^{\prime}(\phi)$ is the first derivative of the Dirac delta function and we have used the relationship $\nabla \delta(\phi)=\delta^{\prime}(\phi) \nabla \phi$. We can further simplify $\delta \mathcal{A}(\phi)$ by using the product rule to make the following substitutions:

$$
\nabla(\delta(\phi)) \cdot \frac{\nabla \phi}{\|\nabla \phi\|}=\nabla \cdot\left(\delta(\phi) \frac{\nabla \phi}{\|\nabla \phi\|}\right)-\delta(\phi) \nabla \cdot\left(\frac{\nabla \phi}{\|\nabla \phi\|}\right)
$$

and

$$
\delta(\phi) \frac{\nabla \phi \cdot \nabla(\delta \phi)}{\|\nabla \phi\|}=\nabla \cdot\left(\delta \phi \delta(\phi) \frac{\nabla \phi}{\|\nabla \phi\|}\right)-\delta \phi \nabla \cdot\left(\delta(\phi) \frac{\nabla \phi}{\|\nabla \phi\|}\right) .
$$

Thus, (10) can be rewritten as

$$
\begin{aligned}
\delta \mathcal{A}(\phi) & =\int_{\Omega}\left[\nabla \cdot\left(\delta \phi \delta(\phi) \frac{\nabla \phi}{\|\nabla \phi\|}\right)-\delta \phi \delta(\phi) \nabla \cdot\left(\frac{\nabla \phi}{\|\nabla \phi\|}\right)\right] d V \\
& =-\int_{\Omega} \delta \phi \delta(\phi) \nabla \cdot\left(\frac{\nabla \phi}{\|\nabla \phi\|}\right) d V,
\end{aligned}
$$

where we have again used phase-periodicity to eliminate the surface integral over the unit cell that arises when applying the divergence theorem to the first integrand. Finally, we may convert $\delta \mathcal{A}$ back into a surface integral over $\Gamma$ by comparing (13) with (8):

$$
\delta \mathcal{A}(\phi)=-\int_{\Gamma}(\nabla \cdot \mathbf{n}) \frac{\delta \phi}{\|\nabla \phi\|} d S .
$$




\subsection{Variation of the volume fraction constraint}

To compute the variation of the volume fraction constraint, we begin by writing the definition of the volume fraction as an integral over the entire unit cell:

$$
f(\phi)=\int_{\Omega_{1}} 1 d V=\int_{\Omega}(1-\Theta(\phi)) d V .
$$

Note that in writing (15), we have implicitly assumed that the volume of the unit cell is 1 . Next, we compute the variation of $f(\phi)$ as follows:

$$
\delta f(\phi)=\int_{\Omega} \delta(1-\Theta(\phi)) d V=-\int_{\Omega} \delta(\phi) \delta \phi d V=-\int_{\Gamma} \frac{\delta \phi}{\|\nabla \phi\|} d S,
$$

where we have again made use of (8) to convert the volume integral over the entire unit cell to a surface integral over $\Gamma$.

\section{Theoretical Analysis of Minimization Problem}

\subsection{Characterization of local extrema}

Using the machinery developed in the previous section, we can characterize the local extrema of the optimization problem (11). A straightforward application of the calculus of variations with constraints leads to the following

Theorem. $\mathcal{A}(\phi)$ is a local extremum subject to the constraint $f(\phi)=f_{o}$ if and only if the surface $\Gamma$ has constant mean curvature.

Proof. $(\Rightarrow)$ Suppose that $\Gamma$ is a local extremum of the total surface area subject to the specified volume fraction constraint. Then it is a local extremum (without constraint) of the Lagrangian [20]:

$$
\mathcal{L}(\phi, \lambda) \equiv \mathcal{A}(\phi)+\lambda\left(f(\phi)-f_{o}\right)
$$

where $\lambda$ is a scalar Lagrange multiplier. Using the expressions for the variation of the surface area and volume fraction derived in the previous section, the total variation of $\mathcal{L}(\phi, \lambda)$ is given by

$$
\begin{aligned}
\delta \mathcal{L}(\phi, \lambda) & =\delta \mathcal{A}(\phi)+\delta\left[\lambda\left(f(\phi)-f_{o}\right)\right] \\
& =-\int_{\Gamma}[(\nabla \cdot \mathbf{n})+\lambda] \frac{\delta \phi}{\|\nabla \phi\|} d S+\left(f(\phi)-f_{o}\right) \delta \lambda .
\end{aligned}
$$

Since this expression must be of zero for any variation in $\phi$ and $\lambda$, (18) implies that

$$
\begin{aligned}
& \nabla \cdot \mathbf{n}+\lambda=0 \text { on } \Gamma \\
& f(\phi)-f_{o}=0 .
\end{aligned}
$$

Equation (20) is just the volume fraction constraint (which is expected and arises for all applications of the method of Lagrange multipliers). Equation (19), however, leads to the conclusion that the mean curvature is constant over the entire surface and is equal to half the Lagrange multiplier. 
$(\Leftarrow)$ Now, suppose that $\Gamma$ is a constant mean curvature surface. Then, the variation in the area simplifies to yield

$$
\delta \mathcal{A}(\phi)=-\int_{\Gamma}(\nabla \cdot \mathbf{n}) \frac{\delta \phi}{\|\nabla \phi\|} d S=-(\nabla \cdot \mathbf{n}) \int_{\Gamma} \frac{\delta \phi}{\|\nabla \phi\|} d S=(\nabla \cdot \mathbf{n}) \delta f(\phi) .
$$

Since any variation of the level set function $\phi$ that does not change the volume fraction must satisfy $\delta f(\phi)=0$, we see that $\delta \mathcal{A}(\phi)=0$ for any volume fraction preserving variation in $\phi$. In other words, $\Gamma$ is a local extremum of the total surface area subject to a volume fraction constraint.

Before moving on, it is worth mentioning some special cases of local extrema of the total surface area with a constrained volume fraction. First, there are the minimal surfaces. Because, they have zero mean curvature, the theorem immediately implies that all minimal surfaces are local extrema of the total surface area under a volume fraction constraint. However, minimal surfaces also have the interesting property that they are local extrema of the total surface area without any constraint. This result follows directly from the expression for the variation in the total surface area because the integrand is identically zero for minimal surfaces. A second class of important examples is the class of constant, nonzero mean curvature surfaces. Two examples are the sphere and infinite cylinder. Both of these objects have a constant, nonzero mean curvature, and thus are local extrema of the total surface area when the volume is constrained. Further examples are provided by any of the nonzero mean curvature surfaces studied by Anderson et al. [10].

These examples demonstrate that the class of minimal surfaces and the class of local extrema of the total surface area under a volume fraction constraint are truly distinct. That surfaces arising from an analysis of local geometric properties differ from those arising when global geometric properties are studied underscores the importance of considering the global geometric features of surfaces. These considerations are especially important from a physical perspective; while the driving force for surface evolution is often local in nature, global constraints (e.g., conserved quantities) are almost always present and can affect the global structures that arise.

\section{Numerical Optimization Procedure}

While Theorem 3.1 provides a theoretical characterization of local minima of the total surface under a constrained volume fraction, it is does not provide a means for obtaining optimal surfaces. In this section, we present a numerical procedure for computing locally optimal surfaces. Our approach follows the work of Osher and Santosa [ 18] which evolves the embedding function $\phi$ along steepest descent directions using the evolution equation:

$$
\delta \phi+v(\mathbf{x})\|\nabla \phi\|=0
$$

with $v(\mathbf{x})$ chosen so that $\phi$ remains in the set of embedding functions that satisfy the volume fraction constraint. As pointed out in [18], this equation is equivalent 
to a Hamilton-Jacobi equation if the change in $\phi$ is viewed as occurring continuously in time. In addition, we use an auxiliary Newton iteration to explicitly enforce the volume fraction constraint when numerical error causes the volume fraction to drift beyond an acceptable tolerance and to generate initial structures that satisfy a prescribed volume fraction constraint [18].

\subsection{Descent direction and projected gradient algorithm}

Following [18, we choose the velocity field $v(\mathbf{x})$ to be the steepest descent direction for the Lagrangian, $\mathcal{L}$. From (18), we know that the variation in $\mathcal{L}$ is given by

$$
\delta \mathcal{L}(\phi, \lambda)=-\int_{\Gamma}[(\nabla \cdot \mathbf{n})+\lambda] \frac{\delta \phi}{\|\nabla \phi\|} d S .
$$

Therefore, choosing $\delta \phi$ such that

$$
\delta \phi=[(\nabla \cdot \mathbf{n})+\lambda]\|\nabla \phi\|
$$

ensures that $\mathcal{L}$ decreases at every iteration. Comparing with (22), we can identify the velocity field $v(\mathbf{x})$ as

$$
v(\mathbf{x})=-[(\nabla \cdot \mathbf{n})+\lambda]
$$

This velocity field has a natural interpretation: the motion of the interface is driven by mean curvature (which tends to shrink the local area of the interface) and the Lagrange multiplier (which tries to keep the volume fraction from changing).

To compute the value of $\lambda$ to use for each iteration, we insist that $\delta \phi$ is chosen to satisfy

$$
\delta f(\phi)=0
$$

Essentially, we are ensuring that $\phi+\delta \phi$ satisfies the constraint equation linearized about the current iteration of $\phi$ :

$$
f(\phi+\delta \phi) \approx f(\phi)+\delta f(\phi)
$$

Substituting (24) into (16) and enforcing (26), we find that the Lagrange multiplier should be set equal to

$$
\lambda=-\frac{\int_{\Gamma}(\nabla \cdot \mathbf{n}) d S}{\int_{\Gamma} 1 d S}=-\frac{\int_{\Gamma}(\nabla \cdot \mathbf{n}) d S}{\mathcal{A}(\phi)} .
$$

Notice that $\lambda$ is equal to twice the average value of the the mean curvature over the surface. 


\subsubsection{Extension of the velocity off of the interface}

The velocity of points off of the zero level set are obtained by extending the velocity on $\Gamma$ in the normal direction [16]. We decided to set the velocity in this way to avoid the mathematical singularities that arise for non-zero level sets with very large mean curvatures when using the "natural" velocity extension ${ }^{2}$. These singularities, which appear at cusps of the embedding function $\phi$, lead to numerical difficulties (e.g., strict stability constraint on the effective time step size).

\subsection{Newton iteration to enforce volume fraction constraint}

Because we are only approximately enforcing the constraint at each time step, the iterates of the embedding function $\phi$ will eventually fail to satisfy the volume fraction constraint. To put an iterate back onto the feasible set after the constraint has been violated by more than an acceptable tolerance, we use a Newton iteration as in [18. We also use the Newton iteration to ensure that the initial condition for the embedding function satisfies the desired volume fraction constraint. For situations where the target volume fraction constraint is far from the actual volume fraction of the initial iterate, we use continuation in $f_{o}$ to improve the convergence of the volume fraction constraint algorithm [21].

We begin by considering the volume fraction of a corrected embedding function: $f\left(\phi^{(0)}+\delta \phi\right)$, where $\phi^{(0)}$ is the uncorrected embedding function. Next, we think of $\delta \phi$ in (24) as a function of $\lambda$ (taken to be an unknown) and introduce a scale factor $\alpha>0$ :

$$
\delta \phi(\lambda)=\alpha[(\nabla \cdot \mathbf{n})+\lambda]\|\nabla \phi\| .
$$

This choice for $\delta \phi(\lambda)$ was originally proposed by Osher and Santosa in [ 18]. While there are certainly other possible choices for $\delta \phi(\lambda)$, we opted to use (29) because it tends to preserve the shape of the surface, it performed well in the present work, and it allowed us to reuse code written for other portions of the computation.

Note that (29) allows us to think of $f\left(\phi^{(0)}+\delta \phi\right)$ as a function of $\lambda$. We can then use a Newton iteration to compute the value of $\lambda$ (and therefore the appropriate correction $\delta \phi$ ) required so that the volume fraction constraint is satisfied: $f\left(\phi^{(0)}+\right.$ $\delta \phi)=0$. It is important to recognize that the choice $\delta \phi$ in the Newton iteration is different from the choice of $\delta \phi$ in the optimization iteration (24). In the latter case, $\lambda$ has a known value and can be explicitly computed; in the former case, $\lambda$ is an unknown variable that is determined through a Newton iteration.

Taking advantage of the level set formulation of our problem, calculating the

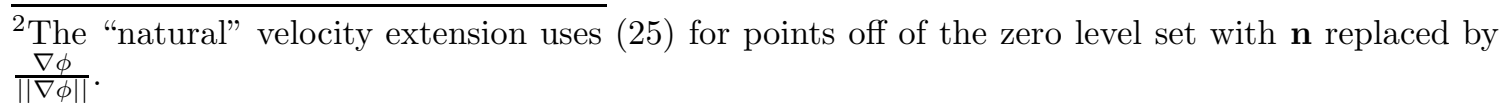


derivative of $f(\lambda)$ with respect to $\lambda$ is straightforward:

$$
\begin{aligned}
D_{\lambda} f\left(\phi^{(0)}+\delta \phi(\lambda)\right) & =-\int_{\Omega} D_{\lambda} H\left(\phi^{(0)}+\delta \phi\right) d V \\
& =-\int_{\Omega} D_{\phi^{(0)}+\delta \phi} H\left(\phi^{(0)}+\delta \phi\right) D_{\lambda}\left(\phi^{(0)}+\delta \phi\right) d V \\
& =-\alpha \int_{\Omega} \delta\left(\phi^{(0)}+\delta \phi\right)\|\nabla \phi\| d V .
\end{aligned}
$$

\subsubsection{Choice of scale factor}

The scale factor is necessary to ensure that the zero level set of $\left(\phi^{(0)}+\delta \phi\right)$ does not vanish and remains within the unit cell; otherwise, the derivative of $f$ with respect $\lambda$ which is computed via as integral over the unit cell in (301) may vanish. Intuitively, the scale factor helps make the discretized version of $\delta \phi$ a reasonable approximation to an infinitesimal variation of $\phi$. Without it, the discrete approximation to $\delta \phi$ would be equal to $[(\nabla \cdot \mathbf{n})+\lambda]$, which may not be small for all Newton iterations. In our simulations, we employed a scaling factor of $\alpha=\left(\min \left\{\Delta x_{1}, \Delta x_{2}, \Delta x_{3}\right\}\right)^{2}$ where $\Delta x_{i}$ is the grid spacing in each $i$-th coordinate direction. This choice for $\alpha$ was selected by numerical experimentation.

\subsection{Numerical implementation issues}

The optimization algorithm based on the algorithmic components discussed in the previous two sections may be summarized in the following steps:

1. Generate an initial configuration for $\phi_{0}(\mathbf{x})$.

2. Evolve the level set function to time $t_{n+1}$ if $\Delta \mathcal{A}_{n}>$ tol.

(a) If necessary, use Newton iteration to enforce the volume fraction constraint.

i. Select an initial guess for $\lambda$.

ii. Repeat the following steps until convergence:

A. Compute $\delta \phi$ using (29).

B. Compute $\phi^{(0)}+\delta \phi$.

C. Update $\lambda$ using: $\lambda^{(k+1)}=\lambda^{(k)}-\frac{f\left(\phi^{(0)}+\delta \phi\left(\lambda^{(k)}\right)\right)-f_{o}}{D_{\lambda} f\left(\phi^{(0)}+\delta \phi\left(\lambda^{(k)}\right)\right)}$ where $(k)$ is a $k$-th Newton iterate.

(b) Compute the Lagrange multiplier $\lambda_{n}$ using (28).

(c) Compute the descent direction $\delta \phi_{n}$ using (24).

(d) Update $\phi$ using $\phi_{n+1}(\mathbf{x})$ to $\phi_{n}(\mathbf{x})+\beta \delta \phi_{n}(\mathbf{x})$. $\beta$ is a scale factor required for stability of the numerical scheme. It is equivalent to a stable time step size when (22) is viewed as a Hamilton-Jacobi equation varying continuously in time.

(e) Periodically reinitialize $\phi(x)$ to an approximate distance function within a sufficiently wide band around the zero level set of $\phi(x)$. 


\subsubsection{Synergy of reinitialization and volume fraction constraint enforce- ment}

Both reinitialization and enforcement of the volume fraction constraint introduce errors into $\phi$. However, the two algorithms often work together in concert - each algorithm reducing the error introduced by the other.

It is well-known that the reinitialization procedure can cause the zero level set of $\phi$ to shift from its "true" position [16, 17]. Fortuitously, the process of enforcing the volume fraction constraint helps to reduce the magnitude of this shift by disallowing large shifts in the zero level set. On the other hand, the volume fraction constraint algorithm can cause a significant shift of the zero level set (especially when generating initial conditions of a specified volume fraction). If a narrow banding type of procedure is used, the zero level set could end up in a region where $\phi$ is not very smooth. Reinitializing in a sufficiently wide band prior to enforcing the volume fraction constraint helps to avoid this problem. Reinitialization after enforcing the volume fraction constraint also helps to keep the zero level set "centered" within the narrow band that is actively updated by the optimization algorithm.

\subsubsection{Stopping criteria for optimization loop}

Because our goal is to minimize $\mathcal{A}$, the change in the interfacial surface area between iterations, $\Delta \mathcal{A}_{n}$ is used as the stopping criteria. Thus, the embedding function, $\phi$, is updated until the $\Delta \mathcal{A}_{n}$ falls below a prescribe tolerance.

\subsubsection{Evaluation of surface integrals}

We numerically evaluate surface integrals by first converting them to volume integrals via (8) and then replacing the delta functions with smoothed approximates. For example, using (8), the Lagrange multiplier (28) may be written in the form

$$
\lambda=-\frac{\int_{\Omega}(\nabla \cdot \mathbf{n}) \delta(\phi)\|\nabla \phi\| d V}{\int_{\Omega} \delta(\phi)\|\nabla \phi\| d V} .
$$

In our computations, we use the following approximation for the $\delta$-function [ 16]:

$$
\delta_{\epsilon}(\phi)= \begin{cases}0 & \|\phi\|>\epsilon \\ \frac{1}{2 \epsilon}\left[1+\cos \left(\frac{\pi\|\phi\|}{\epsilon}\right)\right] & \|\phi\| \leq \epsilon,\end{cases}
$$

where $\epsilon$ is the width of the smoothed $\delta$-function. Following the usual conventions for level set method calculations, we choose $\epsilon=3 \max \left\{\Delta x_{1}, \Delta x_{2}, \Delta x_{3}\right\}$.

\subsubsection{Discretization of the mean curvature}

The discretization of the curvature term is performed by using the natural generalization of the first-order accurate scheme described by Zhao, Chan, Merriman, and Osher $[22]$.

\subsubsection{Grid resolution requirements}

All of our numerical solutions were computed on a unit cube using uniform meshes with sizes between $100 \times 100 \times 100$ and $250 \times 250 \times 250$. For relatively simple surfaces, 
such as the Schwartz P surface, the lowest resolution mesh was sufficient to obtain accurate values for the total surface area $^{3}$. However, complex surfaces, where the distance between separate sheets of the surface is smaller, required higher resolution meshes. For example, in order to obtain results comparable in accuracy to those for the Schwartz P and Schoen G surfaces with a mesh of size $200 \times 200 \times 200$, the Schwartz D surface calculations required a grid of size $250 \times 250 \times 250$. Finer meshes were also required to obtain accurate values for the mean curvature and when finding optimal surfaces for volume fractions other than 0.5 .

\subsubsection{Parallel computation}

Due to the high computational cost (both in time and memory) of the fully three-dimensional shape-optimization calculations, we implemented our algorithm using LSMLIB [ 23], a parallel level set method software library developed by one of the authors. Rapid implementation of our parallel simulation code and LSMLIB was achieved by leveraging the parallel computing framework built into the Structured Adaptive Mesh Refinement Application Infrastructure (SAMRAI) [24, 25] developed at Lawrence Livermore National Laboratory (LLNL).

\section{Numerical Results}

In this section, we present results obtained using the numerical optimization algorithm developed in Section 4. Simulations were run on Linux clusters ${ }^{4}$ using between 2 and 8 nodes depending on the size of the computation. Results were visualized using the VisIt program developed at LLNL.

\subsection{Verification of numerical optimization algorithm}

To verify our numerical optimization algorithm, we tested its ability to accurately compute a few structures whose local optimality are easily verified. These results were computed on a grid of size $100 \times 100 \times 100$.

Our first test case is the cylinder. Because it has constant mean curvature, Theorem 3.1 shows that it is a local minimum of the total surface area under a volume fraction constraint. From symmetry considerations, we expect that any infinitely long channel should evolve towards the locally optimal cylinder structure. In our numerical calculations, we started the optimization algorithm with an infinitely long square channel as the initial surface. As expected, the square channel evolves to the optimal cylindrical channel (see Figure 4).

Our second test case is the sphere. Again, Theorem 3.1 shows it is a locally optimal surface. In this test, we started the optimization algorithm with an closed cube (chosen from symmetry considerations) as the initial surface. Figure 5 shows that the cube evolves to the sphere, as expected.

\footnotetext{
${ }^{3}$ The difference in the total surface area computed at the $100 \times 100 \times 100$ and the $150 \times 150 \times 150$ resolutions was less than $10^{-4}$.

${ }^{4}$ Runs were performed on either a 75 node dual Opteron cluster or a 128 node Intel P4 system.
} 

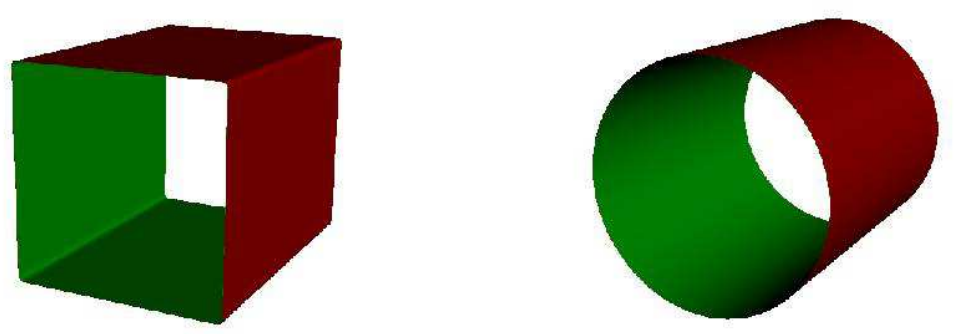

Figure 4. Evolution of an infinitely long square channel (left) to the locally optimal cylindrical channel (right).
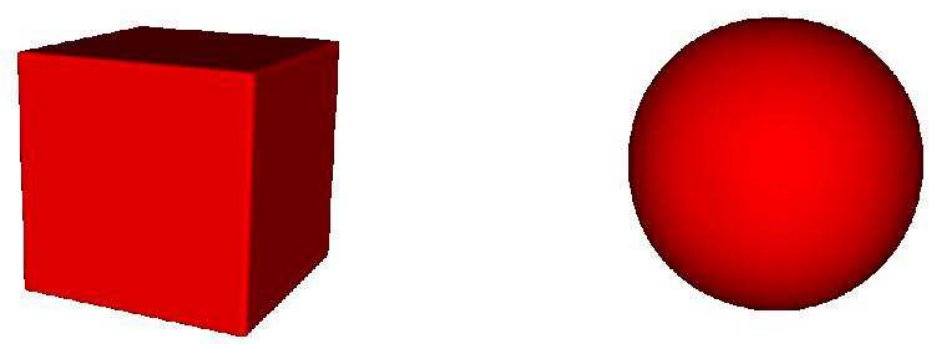

Figure 5. Evolution of a cube surface (left) to the locally optimal sphere surface (right).

In both calculations, the volume fraction constraint algorithm was used to enforce $f_{o}=0.5$. However, the volume fraction did not fluctuate much during the numerical simulations, so the volume fraction constraint algorithm was rarely invoked. Also, notice that for both of these test cases, the level set calculations do not appear to have any difficulty handling sharp edges/corners.

\subsection{Local minimality of Schwartz P, Schwartz D, and Schoen G surface areas}

There are many known ways to compute the Schwartz P, Schwartz D, and Schoen $\mathrm{G}$ triply periodic minimal surfaces. They can be characterized exactly using an Enneper-Weierstrass (complex integration) representation [ 26], generated as the local minima of the scalar order parameter Landau-Ginzburg functional used to describe ordering phenomena in microemulsions [ 27], and approximated by Fourier series using the periodic nodal surface (PNS) expansion [ 28, 2, 29, 30]. It is worth 
pointing out that the approach of minimizing the Landau-Ginzburg functional is actually a phase-field version of our approach for an appropriately chosen form of the energy functional.

Because minimal surfaces have zero mean curvature everywhere, Theorem 3.1 guarantees that the Schwartz P, Schwartz D, and Schoen G surfaces are locally optimal surfaces. However, it does not indicate whether these surfaces are local maxima or minima or saddle points of the total surface area. The numerical optimization procedure developed in Section 4.3 provides a means to answer this question. Since the optimization procedure seeks surfaces with minimal total surface area, locally minimal surfaces should be stable under perturbations. In contrast, local maxima or saddle points of the total surface area should be unstable ${ }^{5}$.
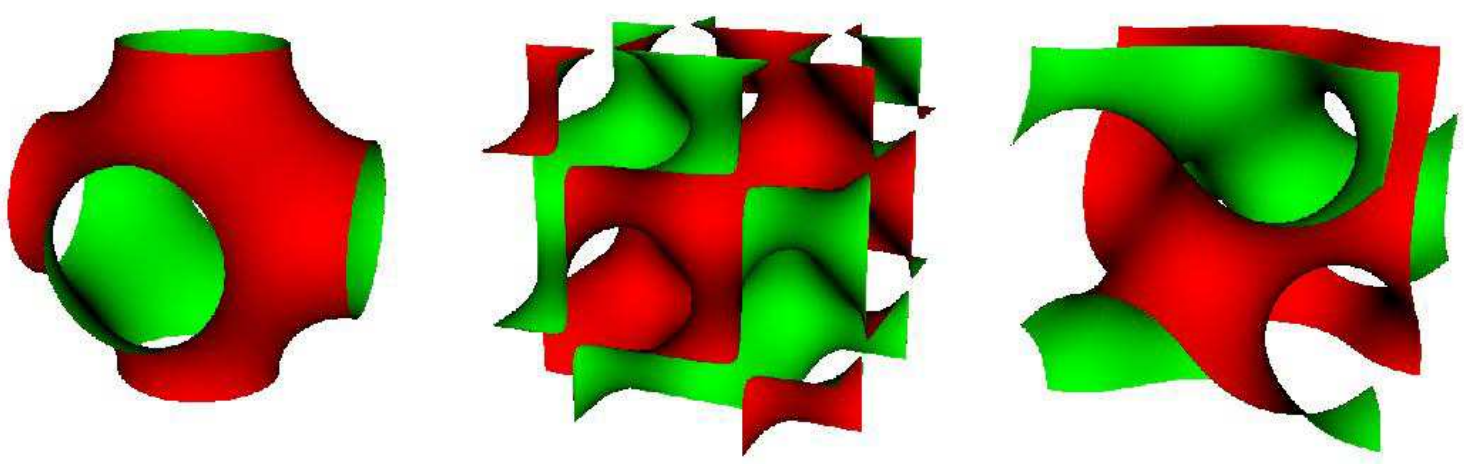

Figure 6. Locally optimal surfaces for $f_{o}=1 / 2$ starting from the PNS approximation to the Schwartz P surface (left), Schwartz D surface (middle), Schoen G surface (right). Notice that the final surfaces are precisely the Schwartz P, Schwartz D, and Schoen G surfaces, which indicates that all three of these surfaces are local minima of the total surface area when the volume fraction is $1 / 2$. These results were computed using a grid of size $150 \times 150 \times 150$.

In our numerical simulations, we start the optimization procedure using the PNS approximations $^{6}$ (which are very good) as the initial $\phi$. As can be seen in Figure [6] the optimization procedure evolves to the correct triply periodic minimal surface. Therefore, we can conclude that the Schwartz P, Schwartz D, and Schoen G surfaces are all local minima of total surface area when $f_{o}=1 / 2$.

\footnotetext{
${ }^{5}$ Note that instability is a necessary by not sufficient condition for a local maximum or saddle point while stability is a sufficient by not necessary condition for a local minimum. Thus, we can only safely draw conclusions when the surface is a local minimum of the total surface area.

${ }^{6}$ In the PNS approximation, each term consists of sine and cosine functions where the high-order terms have a high reciprocal lattice vector norm [29].
} 


\subsubsection{Width of basins of attraction for Schwartz P, Schwartz D and Schoen G}

By starting the optimization procedure with other initial configurations of carefully controlled symmetry, we were able to qualitatively explore the width of the basin of attraction for the Schwartz P, Schwartz D, and Schoen G surfaces.

For the Schwartz P surface, we considered two different initial configurations: (1) triply periodic circular channels (see Figure 7) and (2) $\phi$ set to a function dominated by the second term in the PNS approximation of the Schwartz P surface. As seen in Figure 7 the triply periodic circular channels configurations evolves to the Schwartz $\mathrm{P}$ surface. However, the second initial configuration leads to a new high genus ${ }^{7}$ surface with the same symmetry as the Schwartz P surface (see Figure 8). The symmetry of the computed locally optimal solution is expected from symmetry of the initial configuration, but the genus of the solution is a bit unexpected. Clearly, this result is related to the width of the basin of attraction for the Schwartz $\mathrm{P}$ surface.
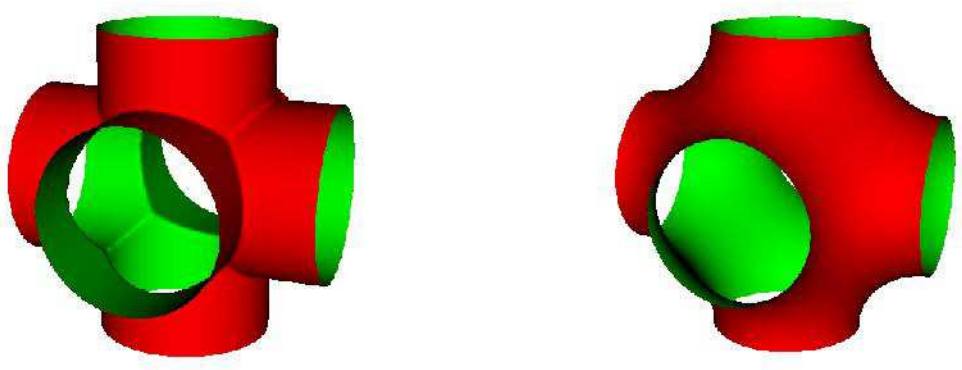

Figure 7. Evolution of triply periodic circular channels (left) to the Schwartz P surface (right).

For the Schwartz D and Schoen G surfaces, we generated initial configurations by setting the initial condition for $\phi$ to be a function dominated by the second term in the PNS approximation of the Schwartz D and Schoen G surfaces, respectively. This procedure allowed us to preserve the symmetry of the of respective surface, while significantly perturbing the actual surface. Figure 9 shows that the Schwartz D surface is the locally optimal structure even if the second term in the PNS approximation is dominant. Even though the initial configuration in Figure 9 looks completely different from the Schwartz D surface, it converges to Schwartz D surface. This result implies that the Schwartz D has a relatively wide basin of attraction. The Schoen G

\footnotetext{
${ }^{7}$ The genus describes how many holes are in a closed surface and therefore is an integer number. In the infinitely periodic case, we describe the genus in a unit cell, which will be a finite number [10].
} 

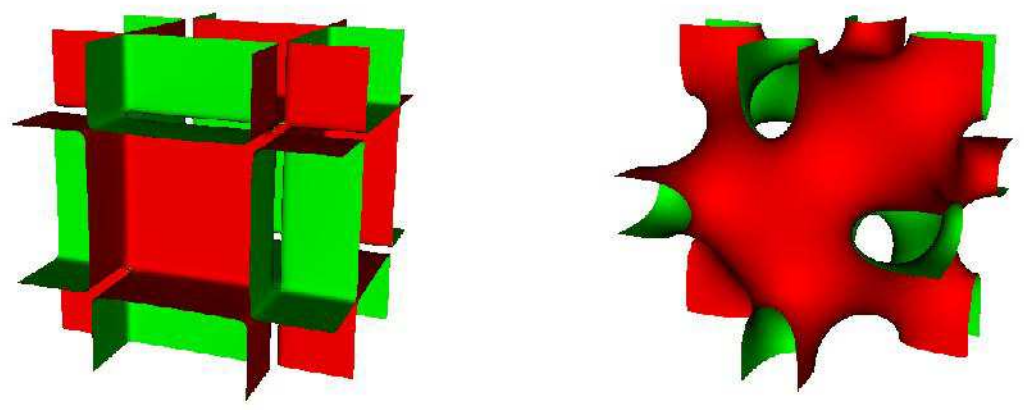

Figure 8. Evolution of a triply periodic surface with the symmetry of the Schwartz $\mathrm{P}$ surface to a new high genus minimal surface (right). The initial structure (left) is obtained by modifying the PNS approximation to the Schwartz P surface so that the second term dominates.

surface shows a similar wide basin of attraction (see Figure 101). It should be noted that the three locally optimal structures all have zero mean curvature at every point on their surfaces.
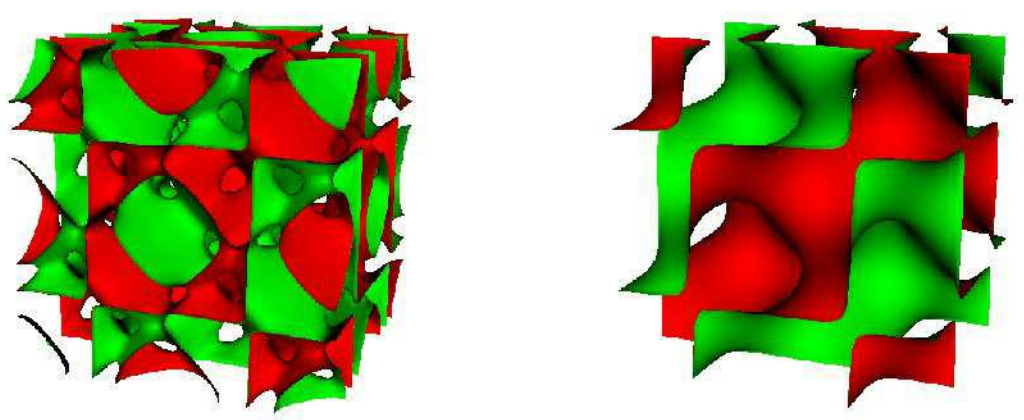

Figure 9. Evolution of a triply periodic surface with the symmetry of the Schwartz D surface to a the Schwartz D surface (right). The initial structure (left) is obtained by modifying the PNS approximation to the Schwartz D surface so that the second term dominates.

\subsection{Locally optimal structures when $f_{o} \neq 1 / 2$.}

In this section, we examine three families of surfaces with the symmetry and topology as the Schwartz P, Schwartz D, and Schoen G surfaces but with different values for the volume fraction of phase 1 . We generated initial configurations with 

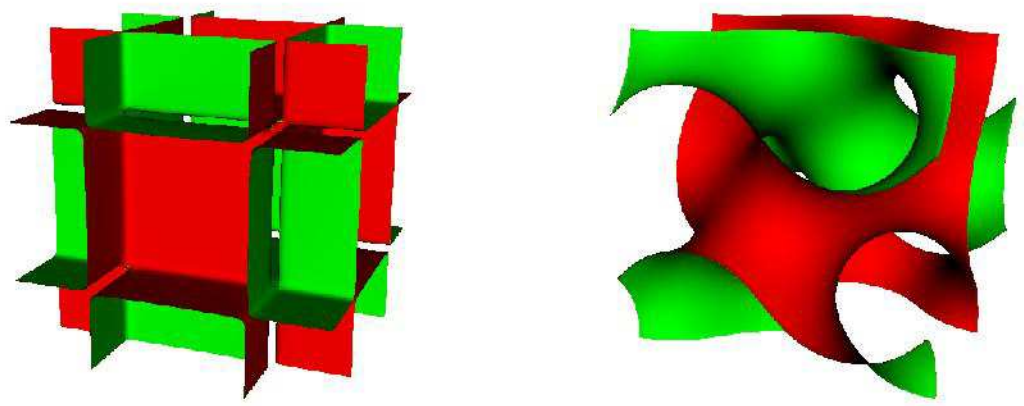

Figure 10. Evolution of a triply periodic surface with the symmetry of the Schoen G surface to a the Schoen G surface (right). The initial structure (left) is obtained by modifying the PNS approximation to the Schoen G surface so that the second term dominates.

the desired symmetry, topology, and volume fractions by taking the PNS Schwartz P, Schwartz D and Schoen G approximations, respectively, and using the volume constraint algorithm to impose the desired volume constraint. We note that to obtain initial conditions for some of the volume fractions reported, we used continuation in the volume fraction to improve the convergence properties of the Newton iteration scheme. These initial configurations were then allowed to evolve to the nearest optimal structure. The total surface area $\mathcal{A}$ is computed using (9) and the mean curvature $H$ is obtained from (31) using the relation $H=0.5 \lambda$. In both calculations, we have used the smoothed delta function, $\delta_{\epsilon}$, defined in (32).

\subsubsection{Schwartz $P$ surface family}

We carried out the shape optimization procedure for the following volume fractions: $0.25,0.3,0.35,0.4,0.45,0.5,0.55,0.6,0.65,0.7,0.75$. Figure 11] shows the six different Schwartz P-type surfaces having specified volume fractions. These calculations were performed on a grid of size $200 \times 200 \times 200$. For a typical simulation, the area stopping criterion $\Delta \mathcal{A}_{n}<10^{-6}$ was reached after approximately 29 hours for a 4 node parallel calculations on the Opteron cluster. The computation time in general increases for volume fractions further from $f_{o}=0.5$ because the volume fraction constraint algorithm is invoked more often.

As the Theorem 3.1 indicates, locally optimal surfaces should have constant mean curvature. Because each surface in the Schwartz $\mathrm{P}$ family is locally optimal, they all constant mean curvature. In Table 1, we report the numerical values of the mean curvature and total surface area for the surfaces in the Schwartz $\mathrm{P}$ surface family that we computed. Figure 12 compares our results with those obtained by Anderson et al. ${ }^{8}$. Note that the data we produce matches the results of Anderson et al. rea-

\footnotetext{
${ }^{8}$ The curves for the data obtained by Anderson et al. are reproduced with the permission of the
} 

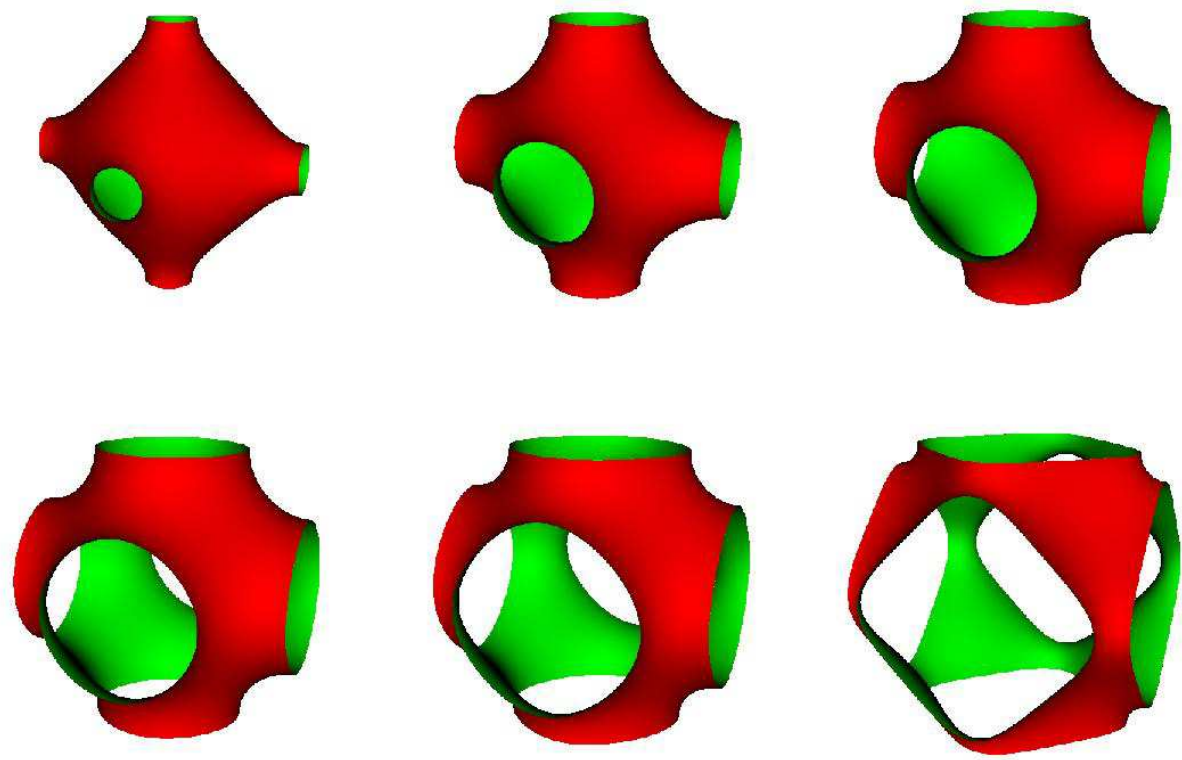

Figure 11. Family of surfaces possessing the symmetry and topology of the Schwartz $\mathrm{P}$ surface but with different volume fractions for phase 1. Upper left to upper right panel: $f_{o}=0.25,0.35,0.45$. Lower left to lower right panel: $f_{o}=0.55,0.65,0.75$.

Table 1

The mean curvature and total surface area of the Schwartz P surface family for different values of the volume fraction.

\begin{tabular}{ccc}
\hline Volume fraction $f_{o}$ & $H$ & $\mathcal{A}$ \\
\hline 0.25 & 1.67 & 2.00 \\
0.3 & 1.12 & 2.14 \\
0.35 & 0.77 & 2.23 \\
0.4 & 0.50 & 2.30 \\
0.45 & 0.24 & 2.33 \\
0.5 & 0.00 & 2.34 \\
0.55 & -0.24 & 2.33 \\
0.6 & -0.49 & 2.30 \\
0.65 & -0.78 & 2.23 \\
0.7 & -1.12 & 2.14 \\
0.75 & -1.67 & 2.00 \\
\hline
\end{tabular}

sonably well. The small discrepancy between the two sets of data is a consequence authors and were generated by digitizing the plots in [10] using the Plot Digitizer program. 
of (1) the lower effective grid resolution of the calculations by Anderson et al. for the Schwartz P surface and (2) the digitization procedure used to extract the curves of Anderson et al. from a printed version of their paper. Figure 12 shows that the volume is almost linearly related to mean curvature in the volume fraction range between $f_{o}=0.35$ and $f_{o}=0.65$. Beyond this range, the mean curvature varies more rapidly as a function of the volume fraction. Anderson's data shows that near volume fractions of 0.75 and 0.25 , the relationship between the volume fraction and mean curvature reverses direction. Due to the limitations of our computational method, we have not yet been able to reproduce the portions of curve between points $\mathrm{B}$ and C. We conjecture that our numerical method fails for these portions of the curves because they are not local minima of the surface area.
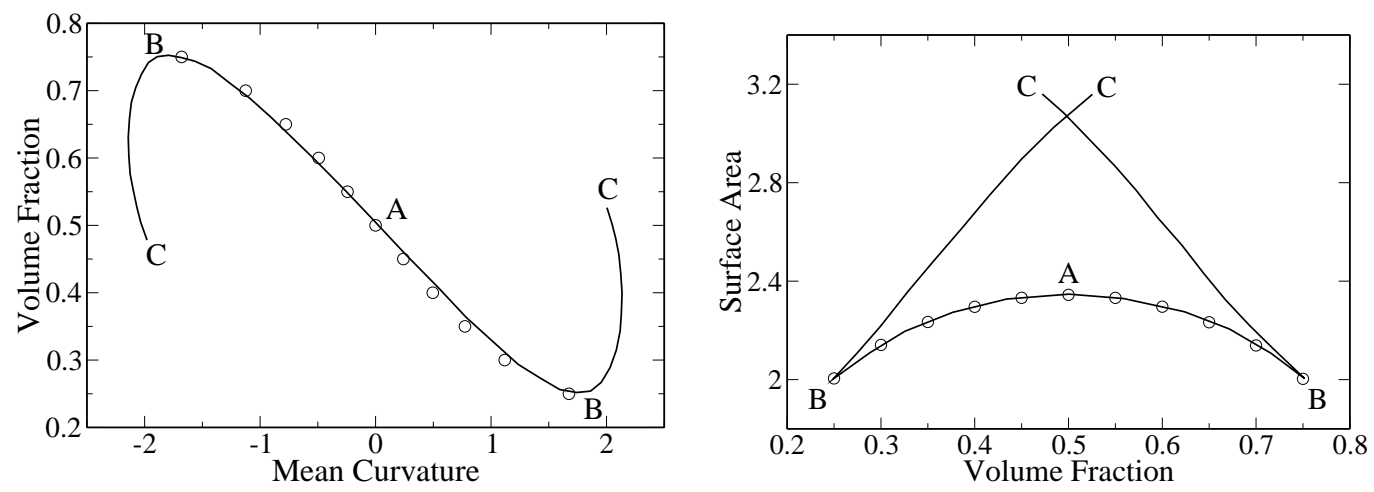

Figure 12. Relationships between the volume fraction, mean curvature, and total surface area for optimal surfaces of the Schwartz $\mathrm{P}$ family: volume fraction versus mean curvature (left) and total area per unit cell versus volume fraction (right). Our results (circles) are compared with those obtained by Anderson et al. (solid lines).

\subsubsection{Schwartz D surface family}

The Schwartz D surface has largest surface area among the three triply periodic surfaces studied in this paper. Since large surface area is inversely correlated with minimum distance between distinct sheets of a surface, the calculations for the Schwartz D surface required higher grid resolution than those for the Schwartz P surface. For the present calculations, we employed a grid of size $250 \times 250 \times 250$. Coarser meshes $($ e.g., $150 \times 150 \times 150)$ failed at extreme values of the volume fractions $\left(e . g ., f_{o}=0.15,0.85\right)$ because the optimal surfaces at these volume fractions have very narrow connections. Due to the larger computational grid, the run time for these simulations took significantly longer than for the Schwartz P-type surfaces. Reaching an area stopping criterion of $\Delta \mathcal{A}_{n}<10^{-5}$ took approximately 30 hours for a 4 node parallel calculation on the Opteron cluster cluster. As with the Schwartz P-type simulations, the running time depended on the volume fraction constraints. 

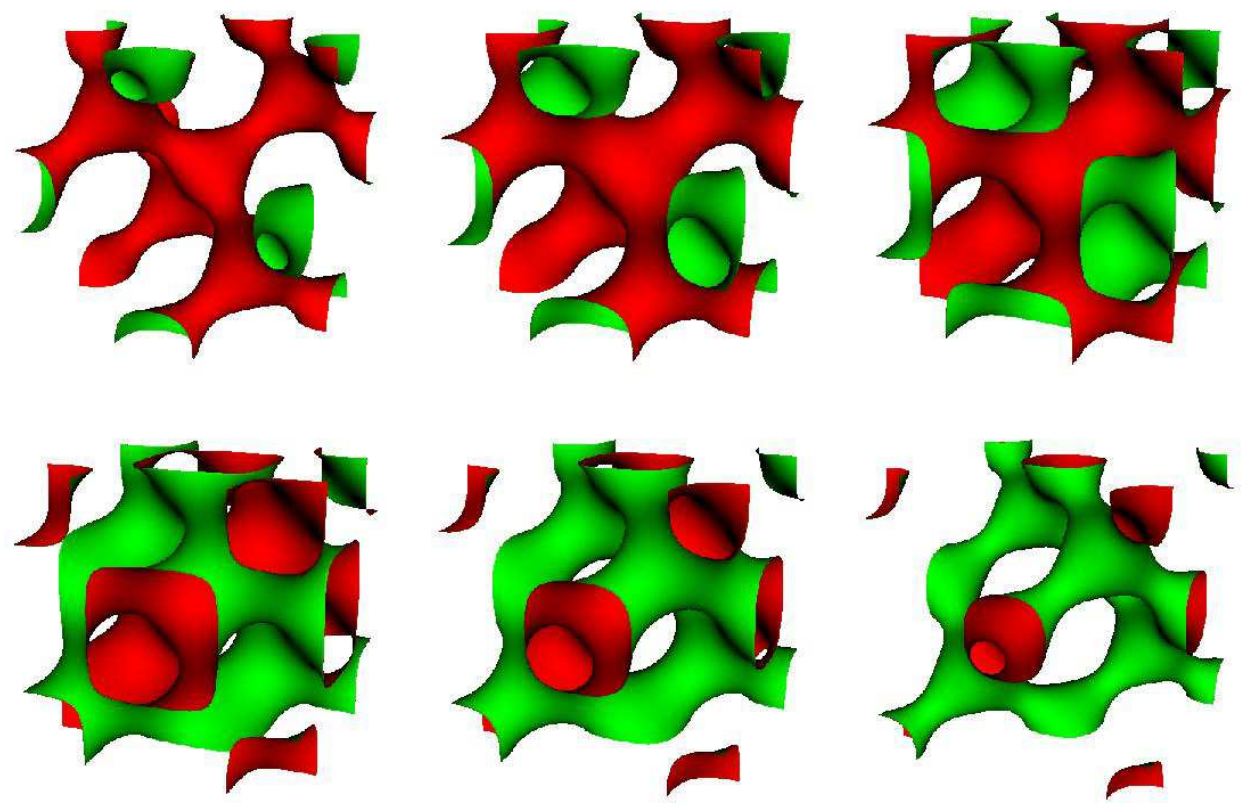

Figure 13. Family of surfaces possessing the symmetry and topology of the Schwartz D surface but with different volume fractions for phase 1. Upper left to upper right panel: $f_{o}=0.15,0.3,0.45$. Lower left to lower right panel: $f_{o}=0.55,0.7,0.85$.

Figure 14 shows excellent agreement with the results of Anderson et al. ${ }^{9}$. However, as with the Schwartz P-type surfaces, our algorithm cannot compute surfaces between points $\mathrm{B}$ and $\mathrm{C}$ on the curve. The numerical values of the mean curvature and total surface area for the surfaces in the Schwartz D surface family are reported in Table 2

\subsubsection{Schoen G surface family}

For these calculations, we employed a grid of size $200 \times 200 \times 200$. Because Anderson et al. did not study Schoen G surfaces, we explored the valid range of volume fractions by stepping the volume fraction in increments of 0.05 from a volume fraction of 0.5 until we observed optimal surfaces that no longer possessed the symmetry of the Schoen G family (Figure [15). We detected the symmetry change (as well as sudden changes in the mean curvature and total surface area) beyond $f_{o}=0.8$ and $f=0.2$. These results were consistent with simulations on finer meshes (e.g., $250 \times 250 \times 250$ ), which leads us to conclude that the $f_{o}=0.8$ and $f_{o}=0.2$ are close to the turning points in the volume fraction versus mean curvature graph (Figure 16).

As shown in Figure 16, the mean curvature range for the Schoen G family of

\footnotetext{
${ }^{9}$ In the calculations of Anderson et al., the effective grid resolution for the Schwartz D surface was higher than for the Schwartz P surface.
} 

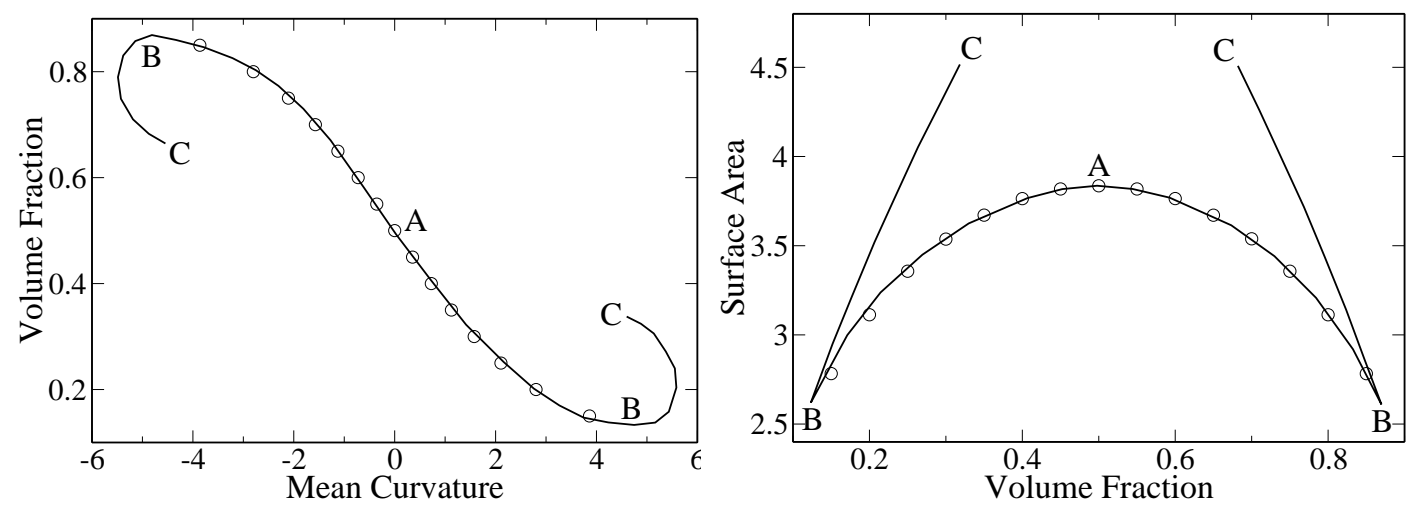

Figure 14. Relationships between the volume fraction, mean curvature, and total surface area for optimal surfaces of the Schwartz D family: volume fraction versus mean curvature (left) and total area per unit cell versus volume fraction (right). Our results (circles) are compared with those obtained by Anderson et al. (solid lines).

Table 2

The mean curvature and total surface area of the Schwartz D surface family for different values of the volume fraction.

\begin{tabular}{ccc}
\hline Volume fraction $f_{o}$ & $H$ & $\mathcal{A}$ \\
\hline 0.15 & 3.86 & 2.78 \\
0.2 & 2.80 & 3.11 \\
0.25 & 2.11 & 3.36 \\
0.3 & 1.58 & 3.54 \\
0.35 & 1.12 & 3.67 \\
0.4 & 0.73 & 3.76 \\
0.45 & 0.35 & 3.82 \\
0.5 & 0.00 & 3.84 \\
0.55 & -0.35 & 3.82 \\
0.6 & -0.72 & 3.76 \\
0.65 & -1.13 & 3.67 \\
0.7 & -1.57 & 3.54 \\
0.75 & -2.10 & 3.36 \\
0.8 & -2.80 & 3.11 \\
0.85 & -3.86 & 2.78 \\
\hline
\end{tabular}

surfaces is similar to that of the Schwartz $\mathrm{P}$ family of surfaces in Section [5.3.1. However, the total surface area of Schoen G-type surfaces are bigger than those of the Schwartz P-type surfaces with the the same volume fractions. Numerical values for the mean curvature and total surface area for surfaces in the Schoen G family 

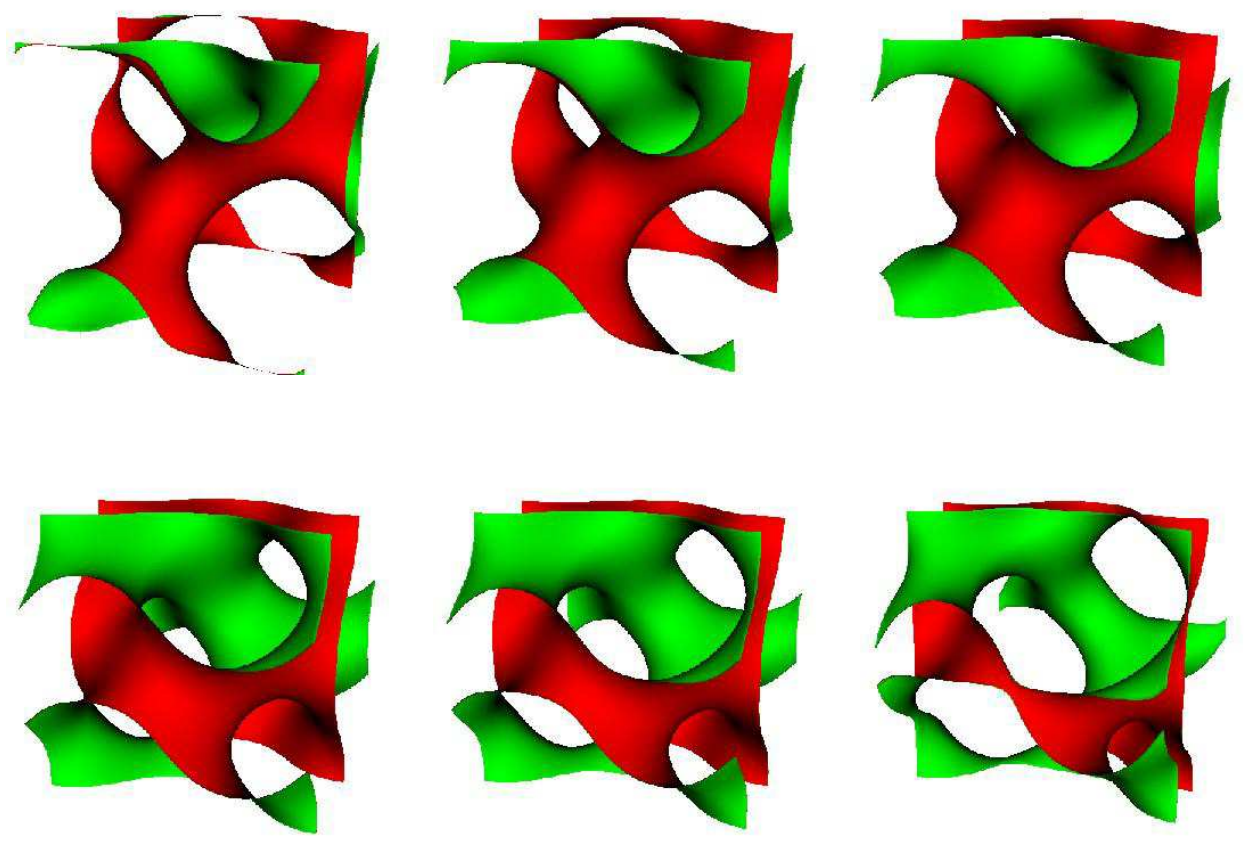

Figure 15. Family of surfaces possessing the symmetry and topology of the Schoen $\mathrm{G}$ surface but with different volume fractions for phase 1. Upper left to upper right panel: $f_{o}=0.2,0.3,0.4$. Lower left to lower right panel: $f_{o}=0.6,0.7,0.8$.

are reported in Table 3
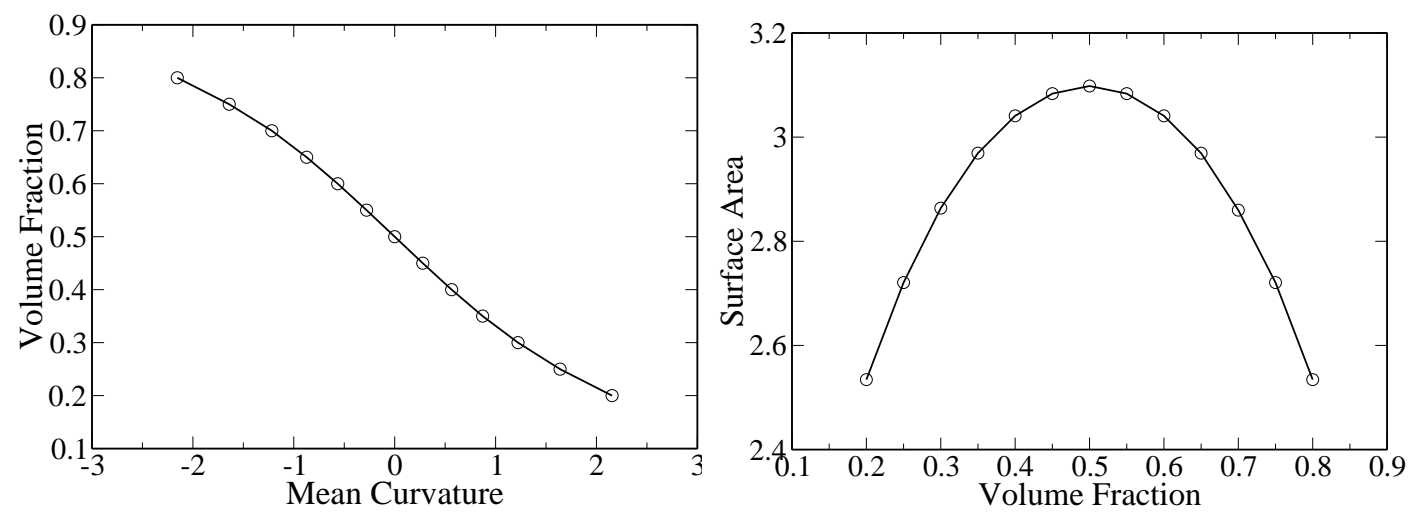

Figure 16. Relationships between the volume fraction, mean curvature, and total surface area for optimal surfaces of the Schoen $G$ family: volume fraction versus mean curvature (left) and total area per unit cell versus volume fraction (right). 
Table 3

The mean curvature and total surface area of the Schoen G surface family for different values of the volume fraction.

\begin{tabular}{ccc}
\hline Volume fraction $f_{o}$ & $H$ & $\mathcal{A}$ \\
\hline 0.2 & 2.15 & 2.53 \\
0.25 & 1.64 & 2.72 \\
0.3 & 1.22 & 2.86 \\
0.35 & 0.87 & 2.97 \\
0.4 & 0.56 & 3.04 \\
0.45 & 0.28 & 3.08 \\
0.5 & 0.00 & 3.10 \\
0.55 & -0.28 & 3.08 \\
0.6 & -0.56 & 3.04 \\
0.65 & -0.87 & 2.97 \\
0.7 & -1.22 & 2.86 \\
0.75 & -1.64 & 2.72 \\
0.8 & -2.15 & 2.53 \\
\hline
\end{tabular}

Following Anderson et al. , it would be interesting to complete the graphs of the volume fraction versus mean curvature and total surface area. However, as with the Schwartz P and D surfaces, we are currently unable to generate surfaces beyond the turning points in the graph of the volume fraction versus mean curvature.

\section{Conclusions}

In this paper, we have generated and probed the structure of the constant mean curvature, triply periodic surfaces (including minimal surfaces) that arise when the total surface area is minimized subject to a constraint on the volume fraction of the regions that the surface separates. Specifically, we have shown that the Schwartz P, Schwartz D, and Schoen G minimal surfaces are local minima of the total surface area under a volume fraction constraint, studied the properties of families of surfaces possessing the symmetry of the Schwartz P, Schwartz D, and Schoen G surfaces at varying volume fractions of the phases separated by the surface, and generated new minimal surfaces (e.g. with high genus) as well as new surfaces with constant, nonzero mean curvature (e.g. family of surfaces with the symmetry of the Schoen $\mathrm{G}$ surface). Unlike many studies on surfaces, our perspective is interesting because it draws attention to the properties of surfaces when global geometric constraints rather than local differential conditions are imposed. Many of our results complement and extend the work by Andersen et al. [10] on nonzero, constant mean curvature surfaces. We emphasize that an important feature of our approach is our ability to easily control the volume fraction of the phases.

The basic approach that we have taken to study optimal triply periodic surfaces has been to use ideas from the level set method and shape optimization. This well- 
known framework has allowed us to formulate the problem in a manner that is amenable both to theoretical analysis and numerical simulation. Theoretically, it allowed us to prove that surfaces with constant mean curvature exactly are precisely the ones that optimize total surface area under a volume fraction constraint on the phases. Computationally, the framework naturally led (via a steepest descent procedure) to a generalization of the two-dimensional shape optimization algorithms presented in [18] and [19]. We note that our discussion of the optimization algorithm provides a more comprehensive description of how variational calculus is used in the context of shape optimization and level set methods. In particular, we demonstrate how the variational formulation of the problem can be used to draw theoretical conclusions (in addition to forming the foundation for the computational method).

Using our numerical algorithm, we explored several optimal triply periodic surfaces. When the volume fractions of the two phases are equal, our theorem implies that the Schwartz P, Schwartz D, and Schoen G surfaces are all local optima of the surface area; numerical simulation shows that these surfaces are actually local minima of the total surface area. For unequal volume fractions of the phases, we partially reproduced the volume fraction versus mean curvature and total surface area versus volume fraction results obtained by Anderson et al. [10] for the Schwartz $\mathrm{P}$ and D surfaces. Unfortunately, due to limitations in our algorithm, we were unable to reproduce the results for extreme values of the mean curvature. We conjecture the surfaces at extreme values of the mean curvature may no longer be local minima of the total surface area. This question certainly deserves require further investigation. In addition to studying the Schwartz $\mathrm{P}$ and Schwartz D families of surfaces, we also extended the work of Anderson et al. by determining the relationship between the volume fraction, mean curvature, and surface area for the Schoen G surface.

One of the original goals our investigation was to show that the triply periodic surface with minimal total surface area at a volume fraction of $1 / 2$ is the Schwartz $\mathrm{P}$ surface. While our numerical simulations provided us with empirical evidence supporting this conjecture, we could not prove these results because our numerical algorithm and theoretical analysis are limited to information about local minima of the total surface area. Thus, the question of the global optimality of the Schwartz P surface remains an open question for future investigation.

\subsection{Future directions}

To close, we mention a few future directions for our work. First, there are research questions that might allow us to gain more insight into the question of global minimality of the Schwartz P surface. For instance, a way to quantify the width of the basin of attractions for the local minima of the total surface area could allow us to be more systematic in our search through the space of triply periodic surfaces (which could be carried out using our numerical optimization algorithm.) Our numerical algorithm could also be used to explore the optimal surfaces possessing a wider array of symmetries.

Second, with a working implementation of our numerical algorithm, we are now in a position to study important questions about the physical properties of two-phase 
materials whose phases are separated by optimal surfaces. For instance, it has been conjectured that microstructures where two phases of unequal volume fraction are separated by a surface that minimize the total surface area lie on the upper bound of effective transport properties for composite materials [ 11, 12]. One obstacle to collecting evidence supporting or refuting this conjecture has been our ability to generate surfaces of minimal surface area. Our optimization algorithm effectively removes this barrier and makes the multifunctional optimization problem for unequal volume fractions more tractable.

\section{Acknowledgments}

YJ and ST gratefully acknowledge the support of the Air Force Office for Scientific Research under grant number F49620-03-1-0406. KTC gratefully acknowledges the support of the National Science Foundation through grant DMR-0502946. The authors thank H. T. Davis for his help in our attempts to obtain the original data for the plots from [10], D. J. Srolovitz for his encouragement and support in the development of the LSMLIB parallel level set method software library, A. Donev for helpful comments on the manuscript, and Bill Wischer for his technical support in carrying out the parallel computations.

\section{REFERENCES}

1. E.A. Lord. Periodic minimal surfaces of cubic symmetry. Current Science, 85(3):346-362, 2003.

2. A.L. Mackay. Crystallographic surfaces. Proc. R. Soc. Lond. A, 442:47-59, 1993.

3. J. Klinowski, A. L. Mackay, and H. Terrones. Curved surfaces in chemical structure. Phil. Trans. R. Soc. Lond. A, 354:1975-1987, 1996.

4. P. D. Olmstead and S. T. Milner. Macromolecules, 31:4011, 1998.

5. L. Yunfeng, Y. Yang, A. Sellinger, M. Lu, J. Huang, H. Fan, R. Haddad, G. Lopez, A. R. Burns, D. Y. Sasaki, J. Shelnutt, and C. J. Brinker. Nature, 410:913, 2001.

6. P. Ziherl and R. D. Kamien. Phys. Rev. Lett., 85:3528, 2000.

7. W. M. Gelbart, A. Ben-Shaul, and eds. D. Roux. Micelles, Membranes, Microemulsions, and Monolayers. Springer Verlag, New York, 1994.

8. T. Landh. From entangled membranes to eclectic morphologies: cubic membranes as subcellular space organizers. FEBS Lett., 369:13-17, 1995.

9. National Research Council. Biomolecular Self-Assembling Materials: Scientific and Technological Frontiers. National Academy Press, Washington, D.C., 1996.

10. D. M. Anderson, H. T. Davis, L. E. Scriven, and J. C. C. Nitsche. Adv. Chem. Phys., 77:337, 1990.

11. S. Torquato, S. Hyun, and A. Donev. Multifunctional composites: Optimizing microstructures for simultaneous transport of heat and electricity. Phys. Rev. Lett., 89:266601:1-4, 2002.

12. S. Torquato, S. Hyun, and A. Donev. Optimal design of manufacturable three- 
dimensional composites with multifunctional characteristics. J. Appl. Phys., 94(9):5748-5755, 2003.

13. S. Torquato and A. Donev. Minimal surfaces and multifunctionality. Proc. $R$. Soc. Lond. A, 460:1849-1856, 2004.

14. Y. Jung and S. Torquato. Fluid permeabilities of triply periodic minimal surfaces. Phys. Rev. E, 72:056319, 2005.

15. H.A. Schwarz. Monatsberichte der koniglichen akademie der wissenscaften zu berlin. Jahrgang, pages 149-153, 1865.

16. S. Osher and R. Fedkiw. Level sets methods and dynamic implicit surfaces. Springer-Verlag, New York, NY, 2003.

17. J.A. Sethian. Level Set Methods and Fast Marching Methods Evolving Interfaces in Computational Geometry, Fluid Mechanics, Computer Vision, and Materials Science. Cambridge University Press, 1999.

18. S.J. Osher and F. Santosa. Level set methods for optimization problems involving geometry and constraints I. Frequencies of a two-density inhomogeneous drum. J. Comp. Phys., 171:272-288, 2001.

19. O. Alexandrov and F. Santosa. A topology-preserving level set method for shape optimization. J. Comp. Phys., 204:121-130, 2005.

20. F. B. Hildebrand. Methods of Applied Mathematics. Prentice-Hall, Inc., 1965.

21. J. P. Boyd. Chebyshev and Fourier Spectral Methods. Dover Publications, Inc., 2001.

22. H.-K. Zhao, T. Chan, B. Merriman, and S. Osher. A variational level set approach to multiphase motion. J. Comput. Phys., pages 179-195, 1996.

23. LSMLIB web site, 2006. http://www.princeton.edu/ ktchu/software/lsmlib/.

24. Richard D. Hornung and Scott R. Kohn. Managing application complexity in the SAMRAI object-oriented framework. Concurrency and Computation: Practice and Experience, 14:347-368, 2002.

25. SAMRAI web site, 2006. http://www.llnl.gov/CASC/SAMRAI/

26. J.C.C. Nitsche. Lectures on Minimal Surfaces, volume 1. Cambridge University Press, Cambridge, 1989.

27. W.T. Góźdź and R. Hołyst. High genus periodic gyroid surfaces of nonpositive Gaussian curvature. Phys. Rev. E, 54(5):5012-5027, 1996.

28. H.G. von Schnering and R. Nesper. Nodal surfaces of Fourier series: Fundamental invariants of structured matter. Z. Phys. B, 83:407-412, 1991.

29. U.S. Schwarz and G. Gompper. Systematic approach to bicontinuous cubic phases in ternary amphiphilic systems. Phys. Rev. E, 59(5):5528-5541, 1999.

30. P.J.F. Gandy, S. Bardhan, A.L.Mackay, and J. Klinowski. Nodal surface approximations to the P, G, D and I-WP triply periodic minimal surfaces. Chem. Phys. Lett., 336:187-195, 2001. 